\title{
Business Impact Künstliche Intelligenz
}

\author{
Benjamin van Giffen • Matthias Knoll
}

(C) Der/die Autor(en) 2020, korrigierte Publikation 2021

KI ist allgegenwärtig - in organisationsinternen Projekten, Produktankündigungen und vor allem in der öffentlichen Diskussion. Praktisch kein Lebens- und Arbeitsbereich bleibt unberührt. Neue Produkte und Dienstleistungen gewinnen durch KI an Attraktivität. Doch in die Euphorie mischen sich Stimmen, die auf die Limitationen, typische Sicherheitsrisiken oder andere reale oder vermutete Gefahren von KI für die Nutzer hinweisen. Und tatsächlich: Wer in die Geschichte der KI zurückblickt, erkennt rasch, dass es über die Jahrzehnte bereits vielfach und in Wellen immer wieder Begeisterung für das Thema Künstliche Intelligenz gab, man aber rasch erkennen musste, dass viele Ansätze noch unvollkommen waren. Häufig lag es nicht zuletzt auch an der verfügbaren Hardware respektive Rechenleistung und der mangelnden Fähigkeit, große Datenmengen zu sammeln, zu strukturieren und in finanziell sinnvollem Rahmen zu speichern.

Das könnte nun erstmals anders sein. Zwar finden sich immer noch kuriose oder gar erschreckende Berichte über unvollkommene, leicht in die Irre zu führende Algorithmen - mit teilweise (lebens-)gefährlichen Folgen. Auch ist nicht überall dort KI enthalten, wo das verkaufsfördernde, weil hochaktuelle KI-Etikett im Rahmen von Marketing-Bemühungen angehängt wird.

Doch es gibt zahlreiche Beispiele, die den Nutzen und die Potentiale von KI zweifelsfrei aufzeigen, etwa in klar abgegrenzten medizinischen Bereichen zur Verbesserung der Diagnostik.

B. van Giffen

Universität St. Gallen, St. Gallen, Schweiz

E-Mail: benjamin.vangiffen@unisg.ch

M. Knoll $(\bowtie)$

Hochschule Darmstadt, Darmstadt, Deutschland

E-Mail: matthias.knoll@h-da.de 
Die vorliegende Ausgabe 331 der HMD greift aus diesem nur schwer zu überblickenden Spektrum von Themen diejenigen Bereiche heraus, die sich in der einen oder anderen Form mit den Auswirkungen der KI auf die Geschäftstätigkeit befassen. Im Fokus stehen daher weniger mathematisch-technische Details. Vielmehr soll dargestellt werden, wie auch und gerade aus Sicht der Führungsebene und der anwendenden Fachabteilungen in Unternehmen mit der Thematik umgegangen werden kann und welche Optionen sich bieten. Managementkonzepte stehen also im Vordergrund.

Die Schwerpunktausgabe beginnt mit einem Einführungsbeitrag, der ein Konzept für das Management von Künstlicher Intelligenz in Unternehmen aufzeigt und strukturiert. Das darin vorgestellte St. Galler Management Modell für Künstliche Intelligenz zeigt sieben Handlungsfelder für den betrieblichen, produktiven KI-Einsatz auf. Der Schwerpunkt des Modells liegt auf den neuartigen betrieblichen und technologischen Herausforderungen, die sich aus den Eigenschaften der KI-Technologie ableiten. Hierzu zählen z. B. Fragestellungen des Managements von KI-Projekten, der Organisation des Betriebs, Regulierung und Compliance, aber auch zum Lebenszyklus-Management von KI-Produkten und Dienstleistungen, einschließlich der Entwicklung und dem Betrieb von KI-Modellen. Neben diesen organisationalen Gestaltungsbereichen sind technologische Fragestellungen zu beantworten, z. B. wie geeignete KI-Infrastrukturen gestaltet werden sollen oder auch wie Cybersicherheit im Umfeld von KI sichergestellt werden kann. Es wird deutlich, dass die Skalierung und Nutzung von KI im produktiven Betrieb über die Entwicklung von KIPrototypen hinausgeht und relativ hohe Anforderungen an das Management dieser vielversprechenden Technologie stellt. Der Einführungsbeitrag liefert hierfür einen umfassenden Einstieg für interessierte Fach- und Führungskräfte.

Nach dieser Einführung widmen sich zwei Folgebeiträge der Wahrnehmung von Technik und Digitalisierung, speziell der KI in der Bevölkerung, sowie der Frage, ob Intelligenztests für KI notwendig (und sinnvoll) sind.

Die sich anschließenden fünf Beiträge entstammen unterschiedlichen Anwendungsbereichen. Der erste Artikel lotet die wirtschaftliche Bewertung von KI in der Praxis aus. Konkreten Branchen- und Tätigkeitsbezug haben die Beiträge zum Einsatz von KI im öffentlichen Sektor sowie im innerbetrieblichen IT-Support. Der Schwerpunkt schließt mit zwei Betrachtungen zum maschinellen Lernen. Diskutiert werden einerseits die Implikationen von KI auf das Datenmanagement in Unternehmen und andererseits der Einsatz von maschinellem Lernen im Rahmen der Kausalanalyse.

Schließlich werfen drei Rezensionen einen Blick auf begleitende Literatur: So erschienen uns die Bücher „Künstliche Intelligenz - Eine Einführung“ von Jerry Kaplan, ,Künstliche Intelligenz für Sales, Marketing und Service“ von Peter Gentsch sowie „Künstliche Intelligenz“ von Peter Buxmann eine gute Ergänzung zu den Themen in dieser Schwerpunktausgabe.

Als Spektrums-Beiträge außerhalb des Schwerpunktes runden eine Diskussion zu agiler Dokumentation und zu Social Intranets als Grundlage für die interne Kommunikation- und Zusammenarbeit die Ausgabe ab. 
Unser herzlicher Dank gilt allen Autorinnen und Autoren, die ihr Wissen mit uns und Ihnen teilen und auf diesem Weg wertvolle Impulse für die Diskussion und Implementierung KI-basierter Lösungen geben.

Wir wünschen Ihnen eine spannende Lektüre, aus der Sie viel Wissen mitnehmen können, und freuen uns über Ihr Lob, aber auch über Ihre Anregungen und Kritik.

\section{Benjamin van Giffen und Matthias Knoll}

Funding Open Access funding enabled and organized by Projekt DEAL.

Open Access Dieser Artikel wird unter der Creative Commons Namensnennung 4.0 International Lizenz veröffentlicht, welche die Nutzung, Vervielfältigung, Bearbeitung, Verbreitung und Wiedergabe in jeglichem Medium und Format erlaubt, sofern Sie den/die ursprünglichen Autor(en) und die Quelle ordnungsgemäß nennen, einen Link zur Creative Commons Lizenz beifügen und angeben, ob Änderungen vorgenommen wurden.

Die in diesem Artikel enthaltenen Bilder und sonstiges Drittmaterial unterliegen ebenfalls der genannten Creative Commons Lizenz, sofern sich aus der Abbildungslegende nichts anderes ergibt. Sofern das betreffende Material nicht unter der genannten Creative Commons Lizenz steht und die betreffende Handlung nicht nach gesetzlichen Vorschriften erlaubt ist, ist für die oben aufgeführten Weiterverwendungen des Materials die Einwilligung des jeweiligen Rechteinhabers einzuholen.

Weitere Details zur Lizenz entnehmen Sie bitte der Lizenzinformation auf http://creativecommons.org/ licenses/by/4.0/deed.de. 